\title{
Le personnage féminin dans les bandes de l'Action catholique (1935-1938)
}

\author{
Sylvain Lemay \\ École multidisciplinaire de l'image \\ Université du Québec en Outaouais \\ Jean-François Boulé \\ École multidisciplinaire de l'image \\ Université du Québec en Outaouais
}

Depuis quelques années, un certain nombre de femmes brillent dans le firmament des auteurs de bande dessinée au Québec. Les signatures d'Iris, Zviane, Julie Rocheleau, Julie Delporte, Cathon, Maryse Dubuc, Isabelle Arsenault, Élise Gravel, Stéphanie Leduc, Sophie Bédard se retrouvent fréquemment sur les couvertures des bandes dessinées québécoises disponibles dans les librairies. Mais ces auteurs sont toujours minoritaires et la bande dessinée demeure encore aujourd'hui associée à un univers masculin. Bien que quelques auteures ont profondément marqué l'histoire de la bande dessinée québécoise telles Sylvie Rancourt et Julie Doucet, la présence des auteurs féminins dans le champ du neuvième art demeure très limitée.

Nous intéressant à l'histoire de la bande dessinée québécoise et travaillant actuellement sur la décennie des années 1930, nous avons eu la curiosité d'interroger la présence féminine dans la bande dessinée au Québec à cette époque ainsi que les particularités des personnages féminins dans les bandes publiées - réalisées par des hommes - dans les quotidiens durant cette décennie.

Nos recherches nous ont permis d'identifier une dessinatrice au Québec qui a œuvré durant les années 1930 : Yvette Lapointe. Cette dernière a publié une série de bandes hebdomadaires intitulée Les petits espiègles dans le quotidien La Patrie du mois de mai au mois d'août 1933. Auparavant, elle avait publié une première bande quotidienne dans L'Illustration en 1932, dont le titre était Pourquoi ? Dans son ouvrage sur l'histoire de la bande dessinée au Québec, Michel Viau la considère comme la première véritable auteure québécoise à signer des bandes dessinées dans un périodique. Elle côtoyait alors, dans les journaux québécois, les bandes d'une auteure américaine, Grace Drayton, dont la bande Pierrot, Clairette et Bouboule était publiée, notamment dans L'Action catholique depuis janvier 1933 
C'est dans ce dernier journal que le corpus sur lequel nous allons nous concentrer ici a été publié. Ce corpus est constitué d'adaptations de romans traditionnels publiés en feuilleton dans différents journaux entre 1935 et 1938. Il n'a jamais été republié par la suite. Dans son ouvrage publié en 2006, et intitulé Un objet culturel non identifié, Thierry Groensteen présentait la bande dessinée comme un art sans mémoire. Il écrit :

C'est un paradoxe: si elle maintient artificiellement en vie certaines séries dont la popularité a su se transmettre d'une génération à l'autre, dans le même temps la bande dessinée est un art qui cultive volontiers l'amnésie et n'a pas grand souci de son patrimoine. (67)

Cela est encore plus vrai en ce qui concerne la bande dessinée québécoise qui, à l'exception des Michel Risque et Red Ketchup de Réal Godbout et Pierre Fournier qui remontent aux années 1980, ne réussit même pas à maintenir en vie des séries importantes de son patrimoine. Toute la production ayant précédé l'entrée de la bande dessinée québécoise dans la modernité au tournant des années 1970 a sombré dans l'oubli. L'amnésie de la bande dessinée québécoise semble presque totale. Il nous faut, par contre, souligner ici quelques initiatives récentes qui tentent de corriger ces lacunes. La Pastèque a notamment republié le livre Retour de vacances de Jacques Gagnier en 2002, une œuvre qui datait de 1946 et Les 400 coups ont intégré dans leur catalogue lors des années 2000, certaines œuvres d'Albert Chartier. Enfin, le nouvel éditeur montréalais Mém9ire a entrepris une vaste publication numérique d'albums de bandes dessinées de la première moitié du vingtième siècle avec sa nouvelle collection «Chronographe ».

La recherche que nous présentons dans le cadre de cet article s'inscrit dans un projet plus vaste et ne présentera que quelques éléments préliminaires d'analyse. Ce projet s'intitule L'Hiver de la bande dessinée québécoise et cherche à mettre en lumière les bandes dessinées réalisées durant La Grande noirceur de Duplesssis en analysant leur caractère idéologique de la majorité tout en les mettant en contraste avec quelques bandes au traitement plus moderne. Cette recherche s'inscrit également dans la poursuite de nos recherches doctorales durant lesquelles nous nous étions intéressés à ce qu'il est convenu d'appeler, suite à un article de Georges Raby publié en 1972, le Printemps de la bande dessinée québécoise, printemps qui a éclos entre 1968 et 1975. Georges Raby identifiait les premières publications du Groupe Chiendent en 1968 comme une éclosion de la production québécoise de bandes dessinées et les années qui allaient suivre comme une période de grande effervescence. Nous retrouvons à ce moment-là, des œuvres modernes, où l'humour et la satire occupent le devant de la scène. Celles-ci contrastaient avec le quasi-monopole des bandes dessinées religieuses publiées aux éditions Fides lors des décennies précédentes. Si Printemps il y a, c'est qu'il devait forcément y avoir eu un Hiver. C'est donc un travail de fouilles 
archéologiques auquel nous nous attelons, fouilles qui bénéficient du travail de défricheur et de précurseur de l'historien de la bande dessinée québécoise, Michel Viau.

Qu'y avait-il comme bande dessinée à cette époque, durant cet hiver de la bande dessinée québécoise ? Tout comme la grande noirceur, cela était-il aussi noir qu'on le prétend ? Quelques noms ont survécu au temps, tels Albert Chartier et Odette Vincent; des titres de revues se sont inscrits dans l'imaginaire collectif, tels François, hérauts et Claire; et si Onésime peut encore prétendre à une certaine reconnaissance à l'intérieur du milieu de la bande dessinée québécoise, il est bien loin de connaître un succès comparable à des Blake et Mortimer, par exemple.

Nous nous intéresserons, pour le moment, aux débuts de notre période, soit les années 1930. Cette décennie a constitué un terreau fertile pour l'éclosion d'œuvres qui sont devenues des classiques de la bande dessinée et ce, tant américaine que franco-belge. C'est durant ces années que se développent les séries Tintin (1929), Tarzan (1929), Buck Rodgers (1929), Mickey Mouse (1930), Dick Tracy (1931), Spirou (1938), etc. Au Québec, par contre, peu de séries pérennes voient le jour durant cette décennie. Le marché québécois est surtout occupé durant ces années par les traductions de bandes américaines. Seuls quelques journaux, dont l'Action catholique vont ouvrir leurs pages à des auteurs locaux au beau milieu d'une pléthore de bandes dessinées américaines.

Le corpus que nous étudions et que nous regroupons sous l'appellation « les bandes catho-nationalistes des années 1930 » été publié dans plusieurs journaux, notamment dans les pages du Devoir. Pour cette étude nous avons utilisé les bandes publiées dans l'Action catholique entre autre parce qu'elles sont publiées avec d'autres bandes d'origine américaine. C'est le 18 juin 1935 que ce journal débute la publication d'un feuilleton en images québécois : L'appel de la race. Il s'agit d'une mise en images du roman de Lionel Groulx originellement publié en 1922 sous le pseudonyme d'Alonié de Lestres. Audessus de la bande, il est indiqué que les légendes sont de Victor Barrette, rédacteur au journal Le Droit et que l'illustrateur est Jules Paquette. Y est également mentionné l'éditeur: L'Association catholique des voyageurs de commerce du Canada, section des Trois-Rivières.

Du 18 juin 1935 au 26 mars 1938, se succéderont, presque sans interruption, de longs récits réalisés par des auteurs québécois. Ce sont onze histoires qui seront publiées, totalisant plus de sept cent bandes. Le plus court, Jean Rivard, comprend trente et une bandes; le plus long, Une de perdue, deux de trouvées, en comprend 126. Il s'agit, pour la majorité, d'adaptations de romans 
québécois du terroir faisant l'apologie des racines canadiennes-françaises et de la religion catholique, tels L'appel de la race de Lionel Groulx. Nous y retrouvons cinq romans contemporains, publiés entre 1922 et 1935 ; trois romans du dixneuvième siècle: Une de perdue, deux de trouvées de Georges Boucher de Boucherville originellement publié en 1849; Jean Rivard d'Antoine Gérin-Lajoie de 1862 et Les anciens canadiens de Philippe Aubert de Gaspé qui date de 1863. Deux récits originaux y seront publiés en 1937, Son chemin de Damas de JeanJacques Cuvelier et Émery Paincourt ainsi que Philippe Beaulieu du même Cuvelier sur un scénario anonyme attribué au Père Gabriel Sarrasin. Enfin, pour clore cette série, nous retrouvons une adaptation du roman américain Le dernier des mohicans de Fennimore Cooper réalisé, encore une fois, par l'illustrateur québécois Jean-Jacques Cuvelier.

Tableau 1 : Les bandes dessinées de l'Action catholique

\begin{tabular}{|c|c|c|c|c|}
\hline Titre & Dates & Bandes & Dessinateur & Scénariste \\
\hline $\begin{array}{l}\text { L'appel de la } \\
\text { race }\end{array}$ & $\begin{array}{l}18 \text { juin au } 9 \\
\text { août } 1935\end{array}$ & 44 & Jules Paquette & $\begin{array}{l}\text { Victor } \\
\text { Barrette }\end{array}$ \\
\hline $\begin{array}{l}\text { La terre } \\
\text { conquérante }\end{array}$ & $\begin{array}{l}10 \text { août au } 4 \\
\text { octobre } 1935\end{array}$ & 46 & James McIssac & $\begin{array}{l}\text { Victor } \\
\text { Barrette }\end{array}$ \\
\hline Jean Rivard & $\begin{array}{l}5 \text { octobre au } \\
11 \text { novembre } \\
1935\end{array}$ & 31 & James McIsaac & $\begin{array}{l}\text { Non } \\
\text { mentionné }\end{array}$ \\
\hline $\begin{array}{l}\text { Une de perdue, } \\
\text { deux de } \\
\text { trouvées }\end{array}$ & $\begin{array}{l}16 \text { décembre } \\
1935 \text { au } 1^{\text {er }} \\
\text { juin } 1936\end{array}$ & 126 & Jules Paquette & $\begin{array}{l}\text { Non } \\
\text { mentionné }\end{array}$ \\
\hline $\begin{array}{l}\text { La campagne } \\
\text { canadienne }\end{array}$ & $\begin{array}{l}13 \text { juin au } 6 \\
\text { août } 1936\end{array}$ & 44 & $\begin{array}{l}\text { Maurice } \\
\text { Raymond }\end{array}$ & $\begin{array}{l}\text { Léovide } \\
\text { Francoeur }\end{array}$ \\
\hline $\begin{array}{l}\text { La ferme des } \\
\text { pins }\end{array}$ & $\begin{array}{l}9 \text { septembre } \\
\text { au } 19 \\
\text { novembre } \\
1936\end{array}$ & 55 & Ernest Sénécal & $\begin{array}{l}\text { Non } \\
\text { mentionné }\end{array}$ \\
\hline $\begin{array}{l}\text { Les anciens } \\
\text { canadiens }\end{array}$ & $\begin{array}{l}24 \text { novembre } \\
1936 \text { au } 4 \\
\text { février } 1937\end{array}$ & 58 & $\begin{array}{l}\text { Jean-Maurice } \\
\text { Massicotte }\end{array}$ & $\begin{array}{l}\text { Pierre } \\
\text { Déléan }\end{array}$ \\
\hline $\begin{array}{l}\text { Pierre } \\
\text { Radisson }\end{array}$ & $\begin{array}{l}6 \text { février au } 7 \\
\text { mai } 1937\end{array}$ & 74 & $\begin{array}{l}\text { Fleurimont } \\
\text { Constantineau }\end{array}$ & $\begin{array}{l}\text { Léovide } \\
\text { Francoeur }\end{array}$ \\
\hline $\begin{array}{l}\text { Son chemin de } \\
\text { Damas }\end{array}$ & $\begin{array}{l}10 \text { mai au } 20 \\
\text { août } 1937\end{array}$ & 87 & $\begin{array}{l}\text { Jean-Jacques } \\
\text { Cuvelier }\end{array}$ & $\begin{array}{l}\text { Emery } \\
\text { Paincourt }\end{array}$ \\
\hline $\begin{array}{l}\text { Philippe } \\
\text { Beaulieu }\end{array}$ & $\begin{array}{l}7 \text { septembre } \\
\text { au } 29 \text { octobre } \\
1937\end{array}$ & 46 & $\begin{array}{l}\text { Jean-Jacques } \\
\text { Cuvelier }\end{array}$ & $\begin{array}{l}\text { Non } \\
\text { mentionné }\end{array}$ \\
\hline
\end{tabular}




\begin{tabular}{|l|l|l|l|l|}
\hline $\begin{array}{l}\text { Le dernier des } \\
\text { Mobicans }\end{array}$ & $\begin{array}{l}3 \text { novembre } \\
1937 \text { au 26 } \\
\text { mars 1938 }\end{array}$ & 100 & $\begin{array}{l}\text { Jean-Jacques } \\
\text { Cuvelier }\end{array}$ & $\begin{array}{l}\text { Non } \\
\text { mentionné }\end{array}$ \\
\hline
\end{tabular}

On le constate aisément avec le choix des romans choisis : il s'agit d'illustrer la grandeur de la race canadienne-française tout en défendant ses valeurs catholiques, le dur labeur, le travail de la terre, le danger des villes, etc.

Les dessinateurs de ces récits sont des illustrateurs dont la signature a déjà pu être observée dans des journaux ou des revues, notamment dans L'oiseau bleu. James McIsaac, par exemple, avait illustré plusieurs des Contes historiques de la Société Saint-Jean-Baptiste durant la décennie précédente. Quant aux scénaristes, nous y retrouvons Victor Barrette, journaliste au Droit qui y a longtemps tenu une chronique s'adressant aux jeunes sous le nom de l'Oncle Jean. Nous y retrouvons également des religieux, Émery Paincourt, curé de Paincourt et ami du Chanoine Groulx.

Évidemment, ce n'est pas tant la qualité de ces bandes qui nous intéresse que l'utilisation d'histoires en images à des fins de propagande. Ces bandes n'ont rien de modernes. La première bande de l'Appel de la race indique bien la teneur de ces histoires en images puisque Victor Barrette y est crédité des légendes. Nous sommes donc en présence d'une suite d'images légendées, ce qui est courant pour les bandes dessinées québécoises de l'époque même si, selon un article de Mira Falardeau, les Québécois ont créé les premières bandes francophones à utiliser systématiquement les phylactères au début du vingtième siècle soit près de vingt années avant Alain Saint-Ogan et ses héros Zig et Puce qui vont débuter leur carrière en France en 1925. Mais au Québec, en 1930, cette forme de narration est associée à la bande dessinée américaine et les valeurs qui y sont véhiculées ne correspondent pas à l'éducation que l'on souhaite pour la jeunesse canadienne-française. Lorsque débute l'Appel de la race, le mardi 18 juin 1935, trois autres bandes sont publiées dans l'Action catholique. Il s'agit de La souris Miquette de Walt Disney, Jacques Vernet de Norman Marah ainsi que de l'Intrépide Georges réalisé par Harry F. Oneil (Broncho Bill). Ces trois bandes utilisent le phylactère. Mais les auteurs québécois des adaptations de romans préfèrent utiliser un mode narratif plus près de l'image d'Épinal, imitant en cela quelque peu Les Contes historiques de la Société Saint-Jean-Baptiste publiés au tournant des années 1920. Ces deux séries d'œuvres ont en commun une utilisation des histoires en images en vue d'éduquer la jeunesse. La mission de ces histoires en images nous parait évidente aujourd'hui, et elle devait également l'être à l'époque. Il n'est pas question ici d'offrir un divertissement, de l'aventure ou de l'humour : il s'agit de convaincre. De convaincre surtout les plus jeunes ; de graver dans leurs jeunes têtes les idées favorables à l'épanouissement de la 
race canadienne-française, ce qu'indique clairement la préface du livre Son chemin de Damas. Écrite par un ancien directeur d'une maison de retraite, elle est adressée au président du comité des retraites fermées des voyageurs de commerce des Trois-Rivières, éditeur de ce qui est appelé ici, des albumimages :

Vos nombreuses publications du même genre, depuis deux ans, accusent chez les vôtres, une activité débordante pour la cause du bien et du beau. Elles vous font grandement honneur et provoquent l'admiration de tous. Soyez-en chaudement félicité et bien sincèrement remerciés... L'enseignement par l'image est le moyen moderne d'atteindre facilement et fortement l'esprit et le cœur d'un grand nombre de gens, trop pressés ou trop paresseux pour suivre une longue dissertation. Les drames en images fixent sans effort l'attention et forment comme instinctivement des convictions. (Cuvelier et Paincourt 11)

Son chemin de Damas et Philippe Beaulieu, les deux derniers récits publiés avant Le dernier des Mobicans, sont les deux seuls scénarios originaux de notre corpus. Ce sont également les deux seuls récits à utiliser systématiquement le phylactère, libérés qu'ils sont du poids de l'adaptation. Ces deux derniers récits ont également en commun d'illustrer les bienfaits des retraites fermées, ces maisons où de jeunes hommes pouvaient s'enfermer quelques jours afin de retrouver les vraies valeurs chrétiennes. Rien d'étonnant à cela puisque l'Association des voyageurs de commerce, section des Trois-Rivières qui édite ces récits est issue de ce mouvement des retraites fermées qui a existé au Canada français à partir de 1911 et qui, dans les années 1930 pouvait se vanter d'avoir accueilli plus de deux cent mille membres.

Nos deux récits originaux illustrent donc les bienfaits des retraites fermées pour les jeunes hommes. Mais ce qui incite les personnages masculins à ces retraites fermées, ce sont les femmes. Dans Son chemin de Damas, Jacques Vincent est manipulé par Dolly Thompson, une Anglaise protestante et par Laurent Martov, présenté comme un «bolcheviste fanatique et fourbe, poltron, ambitieux et perfide, souple et rusé comme un renard, obstiné comme un âne, avec le cynisme du chien et la cruauté du loup ». Quant à Philippe Beaulieu, le mal ne provient pas du communisme ou des Protestants, mais des méfaits de l'alcool. Voulant séduire une jeune femme et ayant raté sa veillée, Philippe va s'encourager avec un verre. La boisson aidant, il devient, pour la jeune femme, beaucoup plus intéressant.

Mais revenons à Son chemin de Damas qui présente un univers presqu'entièrement masculin. Le récit débute avec la présentation des personnages principaux, neuf hommes et trois femmes. Ces trois personnages féminins sont : Dolly, la Protestante, Thérèse la Catholique et Madame Vincent, la mère du personnage principal. On peut voir sur l'image (ill. 1), une certaine 
ressemblance entre les deux jeunes filles, notamment en ce qui concerne la coiffure.

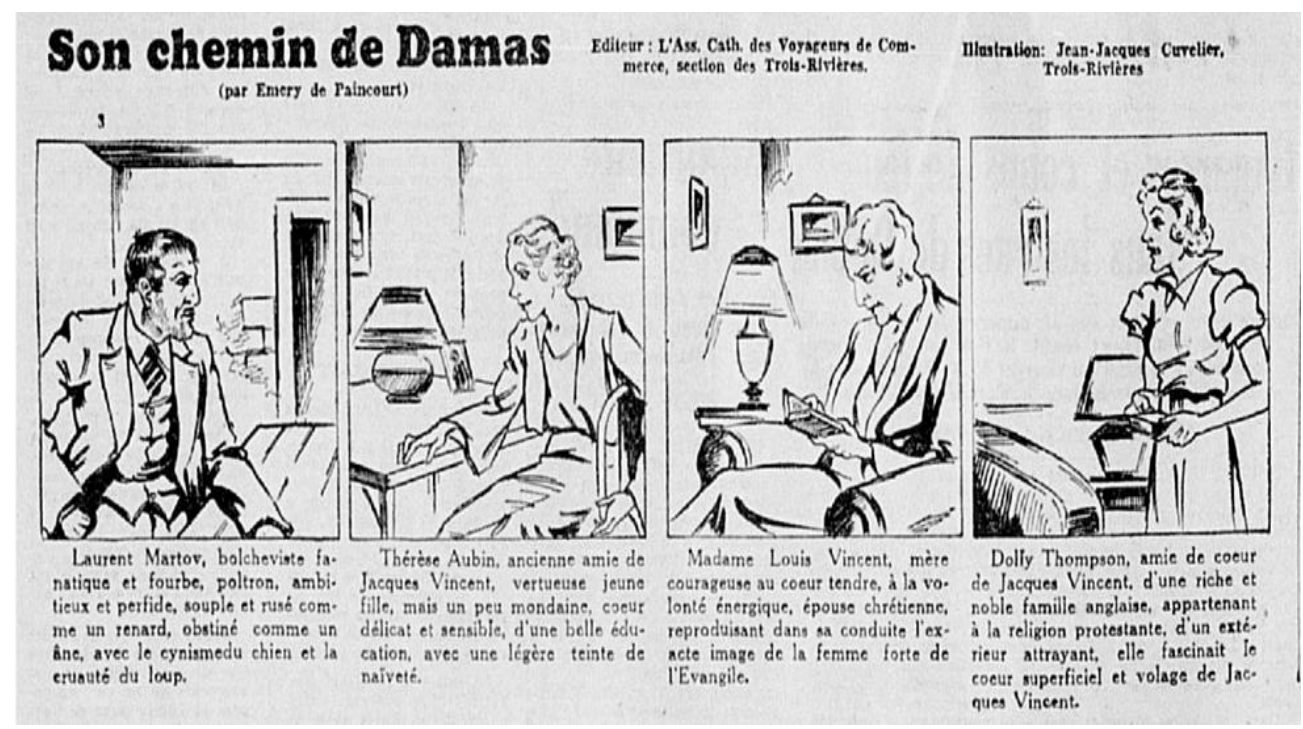

\section{1. L'Action Catholique, 12 mai 1937}

Le rictus de Dolly, par contre, est un indice du danger que représente la jeune anglaise. De plus, elle est debout alors que Thérèse est représentée dans la même position, assise, que « la mère courageuse au cœur tendre, à la volonté énergique, épouse chrétienne, reproduisant dans sa conduite l'exacte image de la femme forte de l'Évangile » (troisième case de l'illustration 1). Quant à Laurent Martov, sa présentation ne laisse aucun doute : avant même de lire qu'il est communiste, nous savons que ce sera le méchant de l'histoire.

Or, étonnamment, à l'intérieur du récit, Laurent Martov ne joue qu'un rôle mineur : il rencontre une fois Laurent (entre les bandes $14^{1}$ et 20 ) et tente de lui faire accepter ses idées révolutionnaires tout en lui demandant son patronage, ce que Laurent fait en lui prêtant de l'argent. Par la suite, il disparaît et ne réapparaît qu'à la bande 78 où il comprend que la retraite fermée de Laurent a fonctionné. Il bat alors lui-même en retraite.

\footnotetext{
${ }^{1}$ Dans l'Action catholique, les bandes ont parfois été présentées dans le désordre. Ainsi, au mois de juin, Dolly quitte Jacques et la semaine suivante elle discute toujours avec lui. Jacques, quant à lui, donne sa démission une journée et fait part de cette même démission dans une bande ultérieure. La bande du 16 juin aurait dû être publiée le 18 juin. La bande publiée le 18 juin aurait dû être publiée le 16 juin ; le 18 juin, on aurait dû publier celle qui du 22 juin; et le 22 juin, nous aurions dû lire celle qui a été publiée le 16 juin.
} 
Pour étudier ces personnages féminins, nous avons regardé du côté d'Annie Pilloy qui, dans son essai Les compagnes des héros de bd publié en 1994 nous offre quelques pistes d'analyse intéressantes. Elle observe les différentes attitudes des personnages de sexe féminin; leurs rôles et métiers; le rapport qui peut exister entre leur apparence physique et leur caractère et la façon dont ils sont considérés par les personnages masculins. Elle recense également le nombre de vignettes où apparaissent des femmes afin d'établir un pourcentage approximatif de ces apparitions. Cette grille d'analyse qui fonctionne assez bien pour l'étude d'un corpus de bandes dessinées modernes demeure toutefois mal adaptée pour un corpus issu de romans à thèse.

Néanmoins, nous sommes donc partis de cette dernière catégorie afin de débuter notre analyse. Une première étude statistique peut ainsi être faite: dans Son chemin de Damas, nous retrouvons 347 cases. Les personnages féminins y sont présents dans 51 d'entre elles, ce qui représente 14,6\% des dessins. Pour Philippe Beaulieu, il s'agit de 32 sur 138, ce qui représente un pourcentage un peu plus élevé de 23,1\%. Par contre, nous n'avons dans cette histoire qu'une seule case occupée uniquement par une femme, sans personnage masculin. Dans Son chemin de Damas, nous avons 5 cases représentant des personnages féminins sans personnages masculins: il s'agit des présentations des trois personnages féminins au début et du personnage de Thérèse en train de prier à la toute fin du récit. Il est clair que nous sommes dans un univers masculin et que le rôle de la femme est celui de soutien aux intérêts des personnages masculins.

Considérons maintenant la représentation qui est faite de ces trois femmes. C'est Dolly, l'Anglaise protestante qui occupe le plus d'espace dans le récit. Elle aura avec Jacques, quatre conversations en tout. Mais nous en savons très peu sur elle. À la bande 16, lors de leur première conversation au chalet, nous apprenons qu'elle désire danser et qu'elle se méfie de Martov (ill. 2).

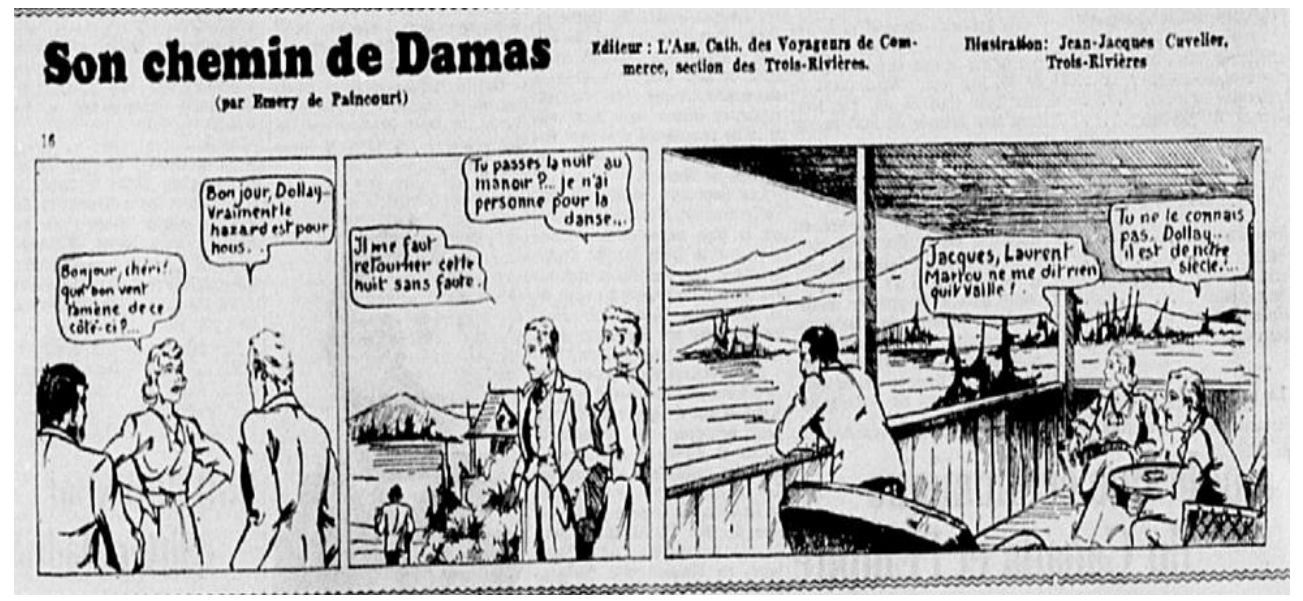




\section{2. L'Action Catholique, 27 mai 1937}

La deuxième conversation (bande 34) se déroule dans le bureau de Jacques où elle offre de l'aider (ill. 3).

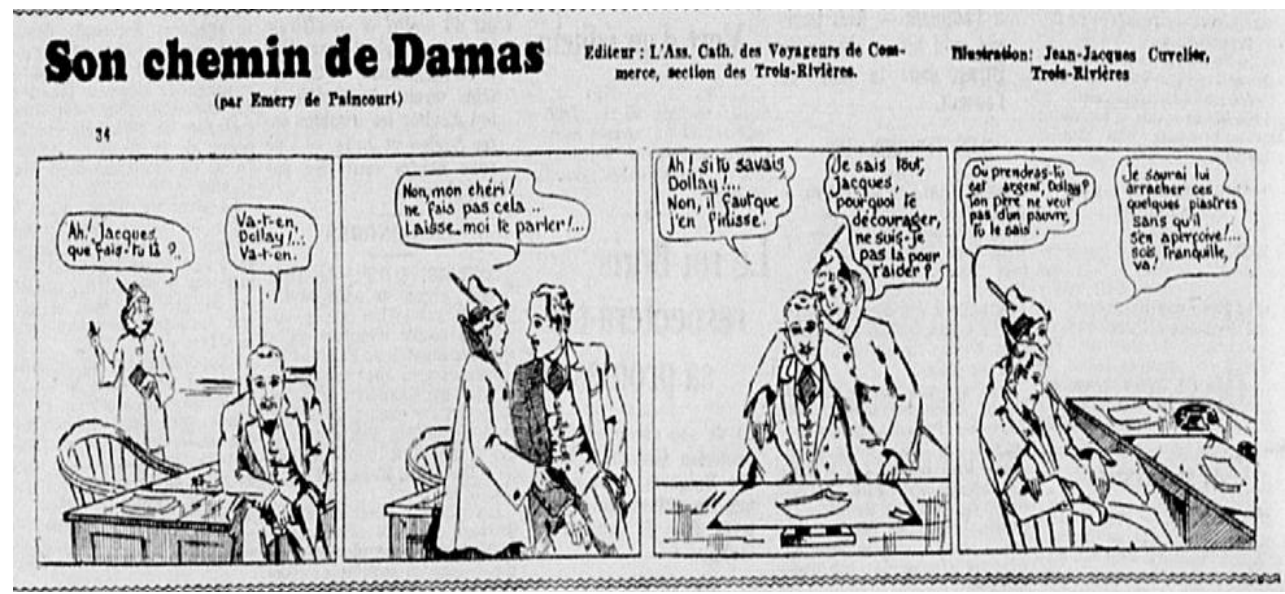

IIl. 3. L'Action Catholique, 17 juin 1937

On réitère ici le fait qu'elle provient d'une riche famille. On voit, par sa posture, que les deux se touchent et qu'elle va jusqu'à envelopper le personnage de Jacques. Une troisième conversation intervient à la bande 39 (ill. 4), où Jacques lui fait part de ses ennuis financiers.

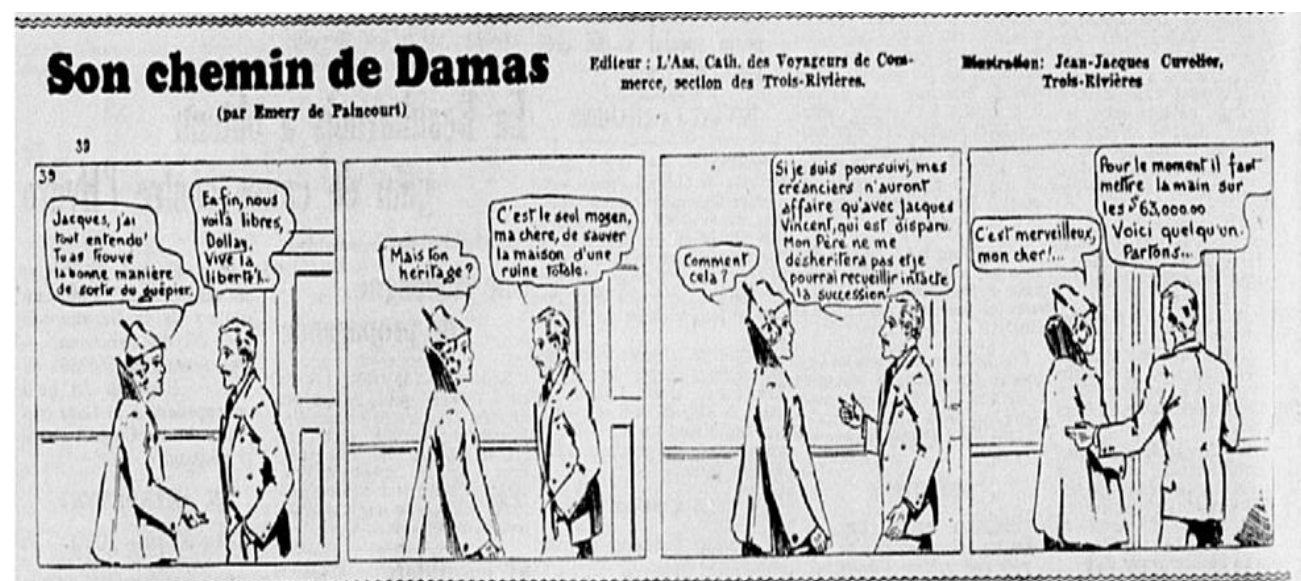

Ill. 4. L'Action Catholique, 23 juin 1937

Puis, le personnage disparaît et ne réapparaît qu'à la bande 72 (ill. 5) pour une dernière conversation où elle comprend, par l'attitude de Jacques, que sa retraite fermée a fonctionné et qu'il n'épousera pas une Protestante. Cette fois, ils ne se touchent plus, le bureau se place entre les deux. Elle quitte la scène 
(nous ne la reverrons plus dessinée) en lançant une menace: «Tu m’infliges l'humiliation d'une trahison. Tu vas apprendre comment un cœur de femme blessé se venge ».

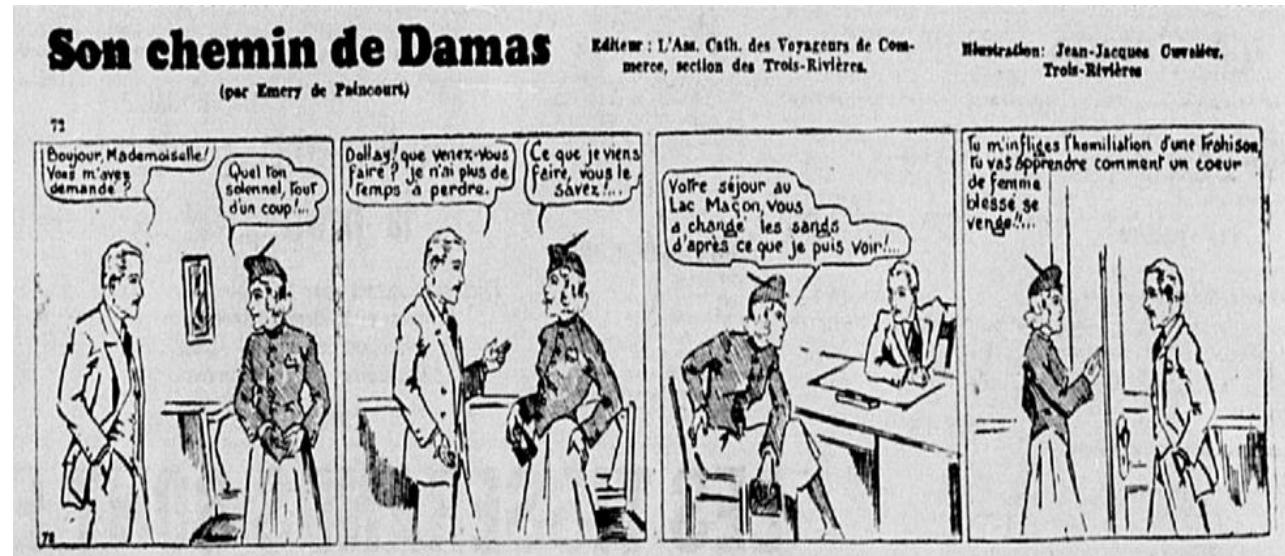

III. 5. L'Action Catholique, 02 août 1937

Présentée en premier au début, Thérèse, qui incarne la vertueuse jeune fille, est absente dans la majorité du récit. Elle apparaît pour la première fois à la bande 78 (ill. 6) après la disparition de Dolly.

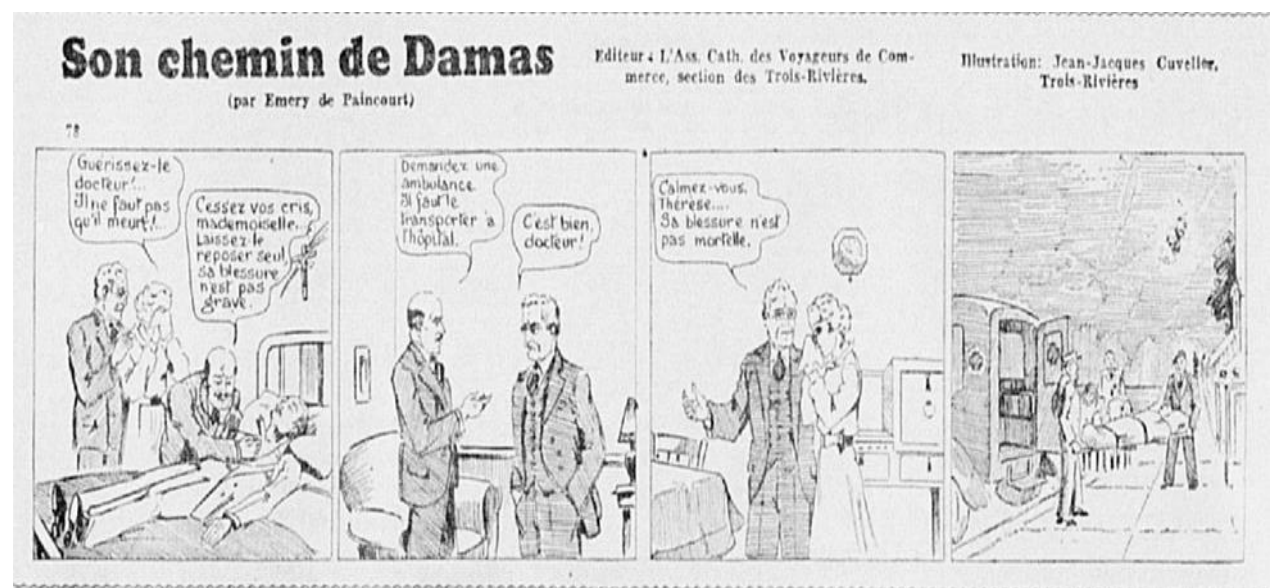

III. 6. L'Action Catholique, 09 août 1937

Jacques est à l'hôpital, nous ne savons pas encore pourquoi, et elle pleure. Elle reviendra revoir Jacques à sa sortie de l'hôpital à la bande 80 (ill. 7). Elle se dit heureuse de le voir guéri, mais elle est exclue rapidement de la conversation. Elle apparaît ensuite à la bande 82 où on laisse sous-entendre qu'une demande en mariage est dans les airs, puis c'est la prière finale de la dernière bande. 


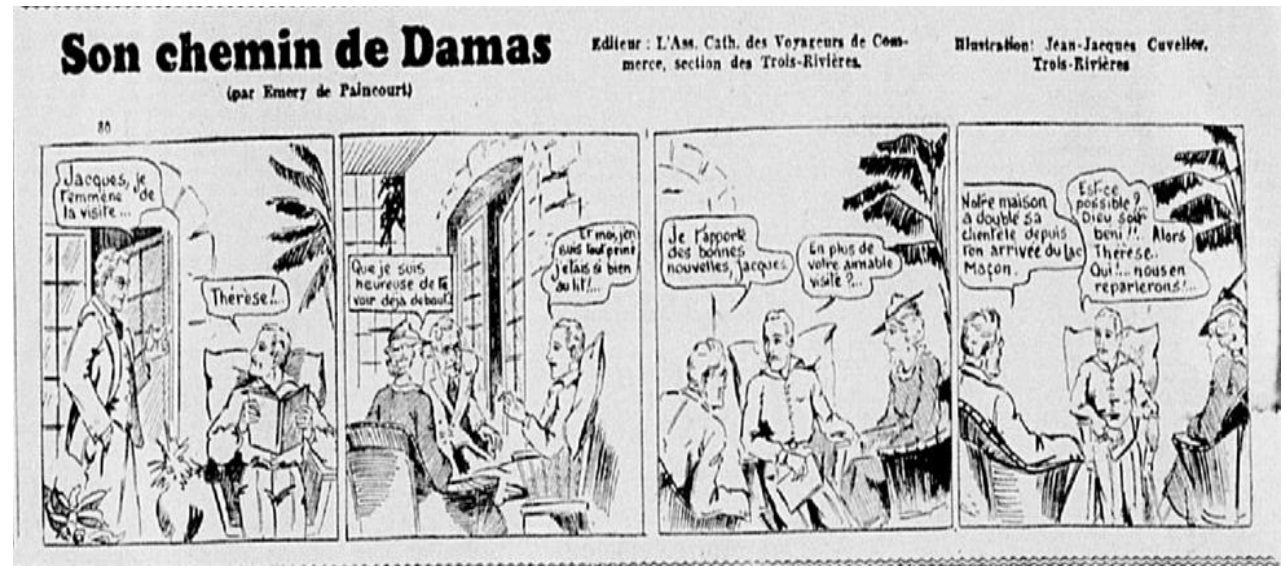

I11. 7. L'Action Catholique, 11 août 1937

La mère, quant à elle, est « l'exacte image de la femme forte de l'Évangile » comme l'indiquait le narrateur dans la troisième case de l'illustration 1. Elle apparait à la bande 69 (ill. 8) pour entendre son fils, sorti vainqueur de sa retraite fermée, lui avouer que ce sont ses prières et ses larmes à elle qui l'ont converti. Stoïque, elle apparaît dans 19 cases vers la fin de l'histoire, 15 de ces cases la représentent assise, solide comme le roc.

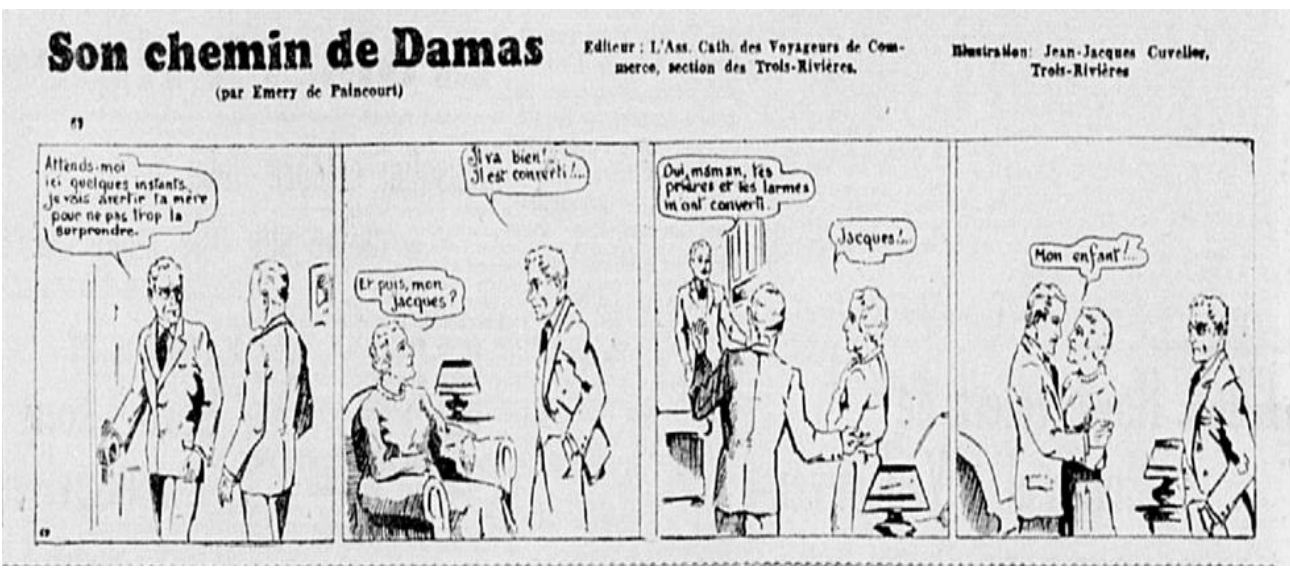

I11. 8. L'Action Catholique, 29 juillet 1937

Il y a donc peu d'action dans ce récit qui met en scène une série de conversations dans des bureaux ou des maisons. Quelques déplacements, en voiture, en canot ou en avion y sont également présents (ill. 9, 10 et 11), mais l'essentiel de l'intrigue est dévoilée à l'intérieur des dialogues. 


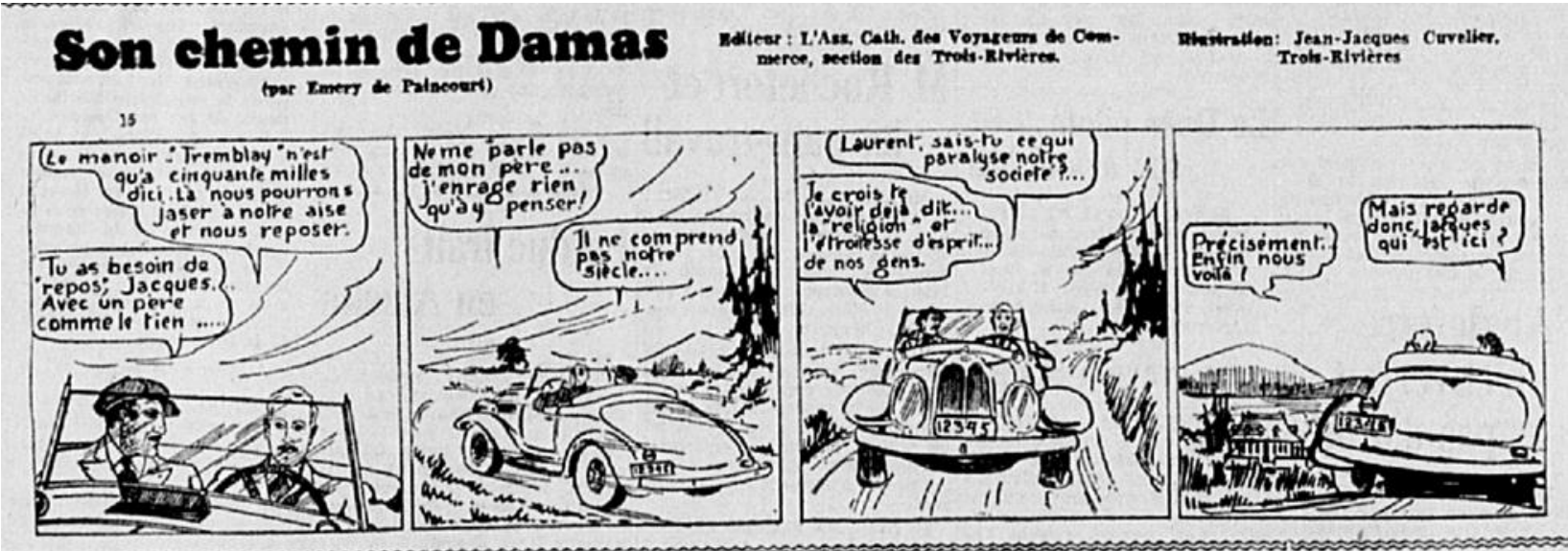

I11. 9. L'Action Catholique, 26 mai 1937

Son chemin de Damas (par Emery de Palneourt)
Zdileur: LAss. Calh. den Vorazeurs de Commerce, sectlion des Trols-pivièrea.
Ninstration: Jean-Jacques Cureller. Trots-Rivieres
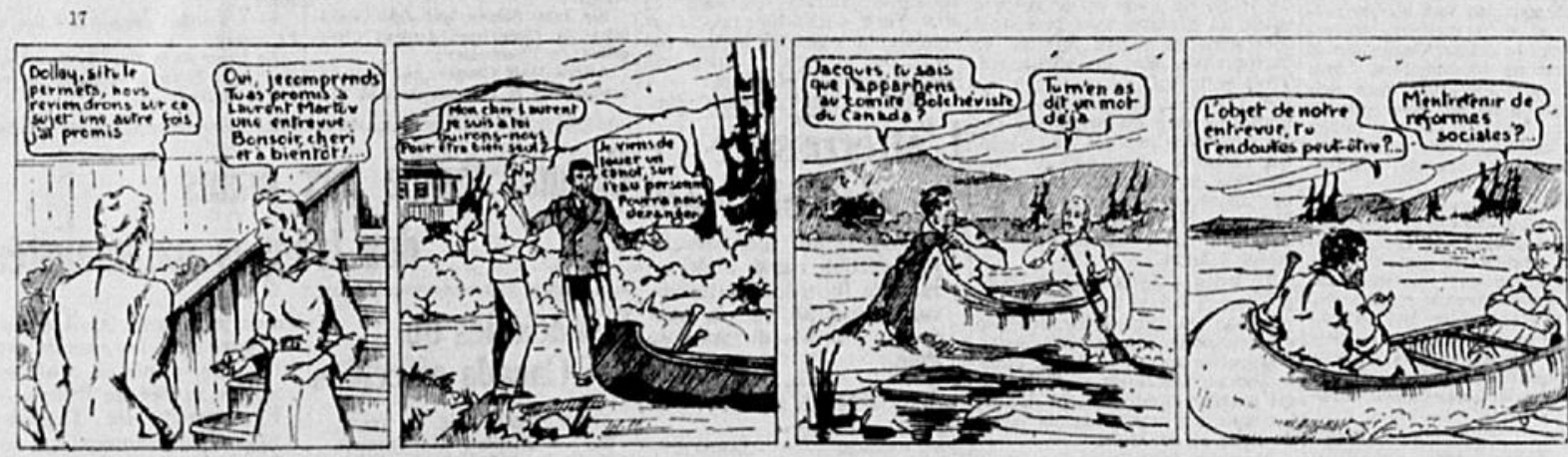

Ill. 10. L'Action Catholique, 28 mai 1937

\section{Son chemin de Damas} (par Emery de Palncouri)
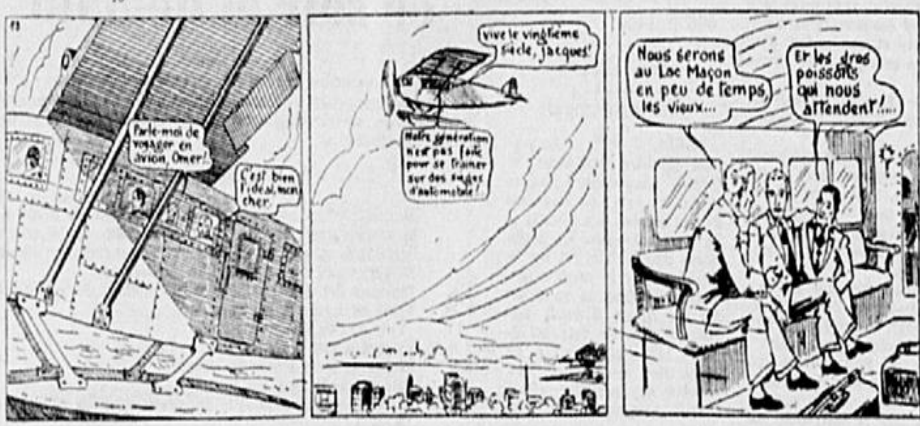

Minsindion: Jeen-Jaceves Cureller.

IIl. 11. L'Action Catholique, 29 juin 1937 
Une seule scène d'action, violente, prend place à l'intérieur du récit et c'est la tentative de meurtre de Jacques. Rien n'est montré, tout se passe en horschamp (ill. 12).

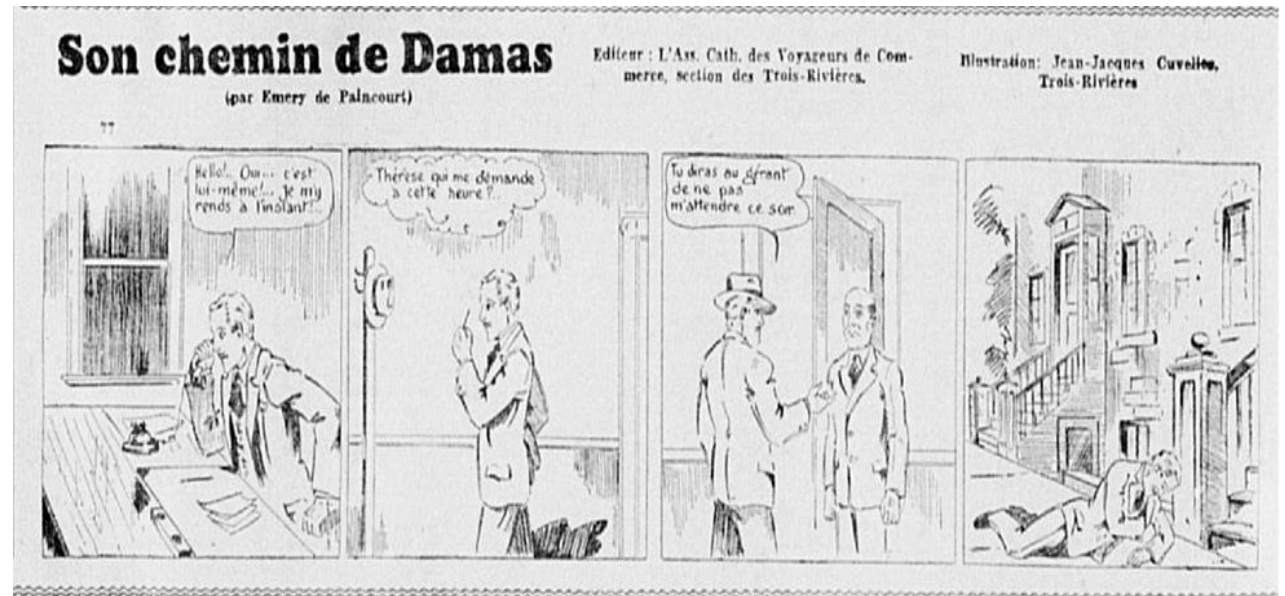

III. 12. L'Action Catholique, 07 août 1937

Si la présentation des personnages pouvait laisser entrevoir qu'une telle action serait posée par le fourbe Laurent Martov, c'est plutôt Dolly, l'Anglaise protestante de riche famille qui, pour se venger de la trahison, va poignarder notre héros. Dolly avait menacé Jacques à la bande 72 et Laurent Martov, l'avait menacé plus légèrement à la bande 76 , c'est-à-dire avant la tentative de meurtre. Nous ne sommes pas dans une enquête policière et le mystère sera expliqué rapidement à la bande 79 , deux jours plus tard par la mère qui raconte tout à son fils (ill. 13) : "Oui mon chéri (Dolly appelait ainsi Jacques)... Tu sais aussi qu'avant de mourir elle a demandé un prêtre catholique ? Tout est donc bien qui finit bien.

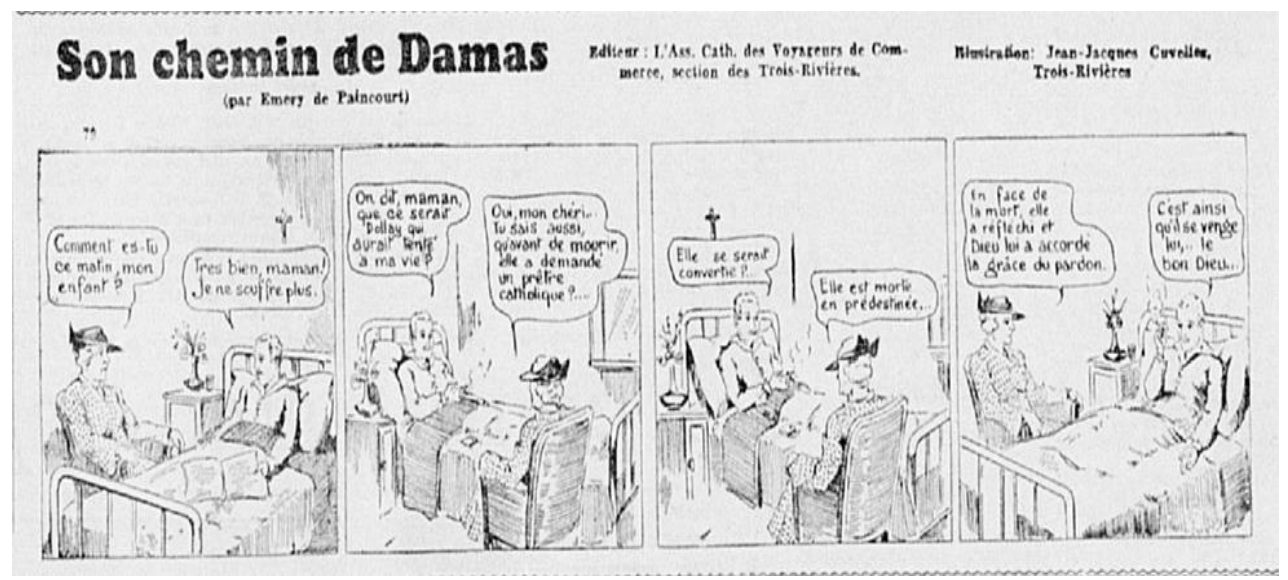

I11. 13. L'Action Catholique, 10 août 1937 
Comme nous l'avons mentionné, cet univers est très masculin : 70 bandes sur 87 ne représentent que des personnages masculins. Dans ces récits, le rôle de la femme est assez clair: la mère pieuse et symbole des valeurs chrétiennes; la jeune femme catholique est à la fois celle qui peut amener l'homme dans le droit chemin et celle qui peut le perdre. Si la menace du communisme est bien établie dès le départ, narrativement et graphiquement, c'est un ennemi plus insidieux que l'on retrouve à l'œuvre, celui des mariages mixtes et de leurs répercussions sur la nation catholique canadienne-française. Son chemin de Damas rejoint en cela la thèse développée par le chanoine Groulx dans le premier récit illustré, l'Appel de la race.

De notre côté, nous n'en sommes qu'au début de ce projet de recherche que nous allons poursuivre durant la prochaine année afin d'en présenter une analyse idéologique plus complète, analyse qui s'inscrira dans la poursuite de notre article publié sur Les contes historiques de la Société Saint-Jean-Baptiste. Mais cette première étude du rôle des personnages féminins à l'œuvre dans ce corpus, nous permettra, nous l'espérons, d'apporter un nouvel éclairage sur les bandes dessinées de cette époque. 


\section{Bibliographie}

Constantineau, Fleurimont et Léovide Francœur. « Pierre Radisson. » L'Action catholique. 6 février au 7 mai 1937.

Cuvelier, Jean-Jacques et Émery de Paincourt. Son chemin de Damas. Montréal : Éditions Mém9ire. 2015. Livre électronique. 76 pages. Publié originellement dans L'Action catholique du 10 mai au 20 août 1937.

Cuvelier, Jean-Jacques. « Philippe Beaulieu.» L'Action catholique. 7 septembre au 29 octobre 1937.

Falardeau, Mira. «La BD française est née au Canada en 1904. » Communication et langages 126 (2000) : 23-46.

Groensteen, Thierry. Un objet culturel non identifié : la bande dessinée. Angoulême : Éditions de l'An 2, 2006.

Lemay, Sylvain, «L'appel de la race ou l'influence des livres. L'exemple des Contes historiques de la Société Saint-Jean-Baptiste de Montréal (19191925). »,Formule Un 1 (2007) : 80-94.

Massicotte, Jean-Maurice et Pierre Déléan. "Les anciens canadiens. » L'Action catholique. 24 novembre 1936 au 4 février 1937.

McIsaac, James et Victor Barrette. « La terre conquérante. » L'Action catholique. 10 août au 4 octobre 1935.

McIsaac, James. « Jean Rivard ». L'Action catholique. 5 octobre au 11 novembre 1935.

Paquette, Jules et Victor Barrette. «L'appel de la race. » L'Action catholique. 18 juin au 9 août 1935.

Paquette, Jules. «Une de perdue, deux de trouvées. » L'Action catholique. 16 décembre 1935 au $1^{\text {er }}$ juin 1936.

Pilloy, Annie. Les compagnes des héros de B.D. : des fermmes et des bulles. Paris: L'Harmattan, 1994.

Raby, Georges. «Le printemps de la bande dessinée québécoise. » Culture vivante 22 (1971) : 12-23.

Raymond, Maurice et Léovide Francœur. «La campagne canadienne. » L'Action catholique. 13 juin au 6 août 1936.

Sénécal, Ernest. «La ferme des pins. »L'Action catholique. 9 septembre 1935 au 19 novembre 1936.

Viau, Michel. Histoire de la bande dessinée au Québec. Tome 1. Des origines à 1979. Montréal : Mém9ire, 2014. 